\title{
Robust Portfolio Selection with Uncertain Exit Time Using Worst-Case VaR Strategy
}

\author{
Dashan Huang* Frank J. Fabozzi ${ }^{\dagger}$ Masao Fukushima ${ }^{\ddagger}$
}

May 9, 2006

\begin{abstract}
In this paper we consider the robust portfolio selection problem involving two types of uncertainties; the uncertainty in the distribution of exit time and the uncertainty in the distribution of portfolio return conditional on exit time. To deal with these uncertainties, we propose a tractable approach by applying worst-case VaR strategy to the case where partial information on the exit time distribution and on the conditional distribution of portfolio return is available, and formulate the corresponding problems as semidefinite programs which can be efficiently solved. Moreover, we present some numerical results with real market data.
\end{abstract}

Keywords: Semidefinite Programming, Worst-Case VaR, Robust Portfolio Selection, Uncertain Exit Time

\section{Introduction}

Black and Litterman (1992), as well as others, have pointed out that for the traditional mean-variance framework, a small perturbation of the inputs may result in a large change in the optimal portfolio. The implication for portfolio managers is that the asset allocation decision is very sensitive to the mean and covariance matrix of return. In turn, this means that the expected return of the component risky assets and the covariance matrix should be sufficiently precise. However, in practice, the expected return and covariance are estimated from data, which are often prone to errors. Thus, because of the lack of robustness, portfolio optimization based on inaccurate estimates impeded the widespread adoption of the mean-variance strategy by practitioners; this also applies to strategies using risk measures other than variance such as value-at-risk (VaR).

Traditional portfolio models are based on the implicit assumption that the investment horizon of an investor is pre-specified, either finite or infinite, and that any investor operates the buy-hold strategy until the explicit exit moment. In fact, as well as taking on asset risk, an investor typically faces exit time risk because the time of his eventual exit upon entering the market is not acknowledged. However, portfolio choice when the investor faces an uncertain exit time - more specifically, how to model the uncertainty of the eventual exit - is a difficult problem to deal with because one must capture the distribution of the asset returns under an uncertain exit time.

Clearly, there are two types of uncertainties in portfolio selection; the uncertainty in the distribution of exit time and the uncertainty in the distribution of portfolio return conditional on exit time. For the

\footnotetext{
*Department of Applied Mathematics and Physics, Graduate School of Informatics, Kyoto University, Kyoto, 606-8501, Japan. Email: dhuang@amp.i.kyoto-u.ac.jp.

†School of Management, Yale University, New Haven, CT06520, USA. Email: frank.fabozzi@yale.edu.

$\ddagger$ Corresponding author, Department of Applied Mathematics and Physics, Graduate School of Informatics, Kyoto University, Kyoto, 606-8501, Japan. Email: fuku@i.kyoto-u.ac.jp. The work of this author is supported in part by the Scientific Research Grant-in-Aid from Japan Society for the Promotion of Science.
} 
second type of uncertainty, Costa and Paiva (2002) and Goldfarb and Iyengar (2003) studied robust portfolio optimization in the mean-variance framework. El Ghaoui et al. (2003) and Zhu and Fukushima (2005) investigated robust portfolio selection using worst-case VaR (WVaR) and worst-case conditional VaR, respectively. For the first type of uncertainty, there is limited academic research on the issue. Martellini and Urošević (2005) were the first to consider the uncertain exit time problem in the traditional mean-variance framework excusively, and found that the portfolio decision is affected not only by exogenous factors but also by endogenous factors.

This paper is motivated by El Ghaoui et al. (2003) and Huang et al. (2006). In contrast to the approach developed by Martellini and Urošević (2005), we propose a WVaR approach, which is extensively discussed in robust portfolio management when the distribution of returns is partially known. We show that it can be extended to the case where only partial information on eventual exit time is given. Thus, our model can incorporate two types of uncertainties; the uncertainty in the distribution of exit time and the uncertainty in the return distribution conditional on exit time, which is different from Huang et al. (2006) where only the uncertainty in exit time is considered.

The remainder of this paper is organized as follows. In Section 2, we discuss the two types of uncertainties when constructing a portfolio with uncertain exit time by means of the original WVaR policy. In Section 3, we generalize the robust optimization technique to deal with the uncertainties. In Section 4, we formulate the portfolio management problem by adopting the generalized WVaR as the measure of risk. Section 5 presents some numerical experiments with real market data. In Section 6, we provide concluding remarks.

\section{Preliminary Problem Formulation}

First, let $r(\boldsymbol{x}, \boldsymbol{y})=\boldsymbol{x}^{\prime} \boldsymbol{y}$ denote the return of a portfolio with decision vector $\boldsymbol{x} \in \mathcal{X} \subseteq \mathcal{R}^{n}$ and random vector $\boldsymbol{y} \in \mathcal{R}^{n}$ that represents the return of individual assets in the portfolio at maturity of the investment horizon $T$. Suppose $\mathrm{E}(|r(\boldsymbol{x}, \boldsymbol{y})|)<+\infty$ for each $\boldsymbol{x} \in \mathcal{X}$. For simplicity of presentation, we assume that $\boldsymbol{y} \in \mathcal{R}^{n}$ has a continuous density function $p(\boldsymbol{y})$. By way of Jorion (2001), all the results can be applied to the case where $p(\boldsymbol{y})$ follows a discontinuous distribution. (For the purpose of clarity, we denote a random variable and its realization by the same symbol, since they can be distinguished clearly from the context.)

In the case where the exit time is uncertain, finding a proper way of specifying the distribution of asset returns $p(\cdot)$ is obviously a difficult task. However, we can decompose the specification into two steps by first specifying the conditional (on exit time) distribution of returns and then determining the distribution of exit time. Just like Huang et al. (2006), the general formula of the unconditional density function can be formulated in this manner: Let $g(\cdot)$ be the density function of exit time $\tau$ and $p_{t}(\cdot)$ be the conditional (on exit time $t$ ) density function of the asset returns. Then the corresponding unconditional density function is given by

$$
p(\cdot)=\int_{0}^{T} p_{t}(\cdot) g(t) d t .
$$

In particular, if the exit time $\tau$ follows a discrete distribution on time $\left\{t_{1}, t_{2}, \cdots, t_{m}\right\}$ with $\operatorname{Pr}\left(\tau=t_{i}\right)=\lambda_{i}$, $\sum_{i=1}^{m} \lambda_{i}=1, \lambda_{i} \geq 0, i=1, \cdots, m$, then we have

$$
p(\cdot)=\sum_{i=1}^{m} \lambda_{i} p_{i}(\cdot),
$$

where we denote $p_{i}(\cdot)$ for $p_{t_{i}}(\cdot)$ in the discrete case throughout for brevity.

From a practical point of view, we deal with a discrete version of the probability distribution of $\tau$ to develop the model. The reason is not only that this treatment will result in a tractable model, but also that it meets the practical purpose since the allowable exiting moment in reality is discrete. Then, by (1), we can 
obtain the unconditional mean $\overline{\boldsymbol{y}}$ and covariance $\Gamma$ of portfolio return immediately as

$$
\begin{aligned}
\overline{\boldsymbol{y}} & =\int_{\boldsymbol{y} \in \mathcal{R}^{n}} \boldsymbol{y} p(\boldsymbol{y}) d \boldsymbol{y}=\sum_{i=1}^{m} \lambda_{i} \overline{\boldsymbol{y}}_{i}, \\
\Gamma & =\int_{\boldsymbol{y} \in \mathcal{R}^{n}}(\boldsymbol{y}-\overline{\boldsymbol{y}})(\boldsymbol{y}-\overline{\boldsymbol{y}})^{\prime} p(\boldsymbol{y}) d \boldsymbol{y}=\sum_{i=1}^{m} \lambda_{i} \Gamma_{i},
\end{aligned}
$$

where $\overline{\boldsymbol{y}}_{i}$ and $\Gamma_{i}$ are the conditional mean and covariance of portfolio return exiting at time $\tau=t_{i}$.

According to El Ghaoui et al. (2003), for a given probability level $\epsilon \in(0,1]$, if the actual values of $\overline{\boldsymbol{y}}$ and $\Gamma$ are known with certainty, we get the following optimization problem of portfolio selection via minimizing WVaR (here WVaR means the worst-case VaR subject to all probability distributions of portfolio return with the common $\overline{\boldsymbol{y}}$ and $\Gamma$ ):

$$
\begin{aligned}
\min _{(\boldsymbol{x}, \gamma, \varsigma, \Delta) \in \mathcal{R}^{n} \times \mathcal{R} \times \mathcal{R} \times \mathcal{S}^{n}} & \gamma \\
\text { s.t. } & \langle\Delta, \Gamma\rangle-\boldsymbol{x}^{\prime} \overline{\boldsymbol{y}}+\kappa(\epsilon)^{2} \varsigma \leq \gamma, \\
& \left(\begin{array}{cc}
\Delta & \boldsymbol{x} / 2 \\
\boldsymbol{x}^{\prime} / 2 & \varsigma
\end{array}\right) \succeq 0, \quad \boldsymbol{x} \in \mathcal{X},
\end{aligned}
$$

where $\varsigma \in \mathcal{R}, \Delta \in \mathcal{S}^{n}, \mathcal{S}^{n}$ denotes the space of $n \times n$ symmetric matrices, $\langle\Delta, \Gamma\rangle=\operatorname{Tr}(\Delta \Gamma)$ denotes the standard scalar product in $\mathcal{S}^{n}$, and $\kappa(\epsilon)=\sqrt{\frac{1-\epsilon}{\epsilon}}$. Here $\kappa(\epsilon)$ is an upper bound of the $\epsilon$-quantile of the portfolio return with the probability $\epsilon$ that the portfolio loss $-r(\boldsymbol{x}, \boldsymbol{y})$ exceeds $\gamma$ (see Bertsimas and Popescu (2005) and El Ghaoui et al. (2003) for details). For two symmetric matrices $A, B \in \mathcal{S}^{n}, A \succeq B$ (resp. $A \succ B$ ) means $A-B$ is positive semidefinite (resp. definite). In this paper we assume $\Gamma \succ 0$, i.e., there are no redundant assets in the portfolio. $\mathcal{X}$ is specified by a set of constraints including budget constraints, target return constraints, regulatory constraints, and so on.

Although (4) has a second-order-cone counterpart which is more popular in robust optimization, in this paper we use the semidefinite formulation of WVaR because this formulation can be easily extended to incorporate the uncertainty in eventual exit time, as shown later. More specifically, using (2) and (3), we have

$$
\begin{aligned}
\min _{(\boldsymbol{x}, \gamma, \varsigma, \Delta) \in \mathcal{R}^{n} \times \mathcal{R} \times \mathcal{R} \times \mathcal{S}^{n}} & \gamma \\
\text { s.t. } & \sum_{i=1}^{m} \lambda_{i}\left[\left\langle\Delta, \Gamma_{i}\right\rangle-\boldsymbol{x}^{\prime} \overline{\boldsymbol{y}}_{i}\right]+\kappa(\epsilon)^{2} \varsigma \leq \gamma, \\
& \left(\begin{array}{cc}
\Delta & \boldsymbol{x} / 2 \\
\boldsymbol{x}^{\prime} / 2 & \varsigma
\end{array}\right) \succeq 0, \boldsymbol{x} \in \mathcal{X} .
\end{aligned}
$$

Note that in the special case where $m=1$, problem (5) reduces to the original WVaR minimization problem.

It is easy to see that problem (5) is a semidefinite program, which would be efficiently solved if the inputs $\lambda_{i}, \overline{\boldsymbol{y}}_{i}$ and $\Gamma_{i}$ are well determined. However, they are usually generated from estimation of some data, and hence suffer from inaccurate data and estimation errors, which implies that we cannot obtain their actual values in practice. On the other hand, we have certain confidence that the actual values are not far from the estimated values. Thus, if it is difficult to obtain the precise parameter values, an intuitive alternative approach is to generalize it by assuming the inputs only known to belong to a given convex set via robust optimization, a useful technique developed in the past decade. 


\section{Robust Problem Formulation}

Based on the above analysis, we assume that the probability vector of exit time and the conditional expected return and covariance matrix lie in some specified sets containing almost all the possible scenarios:

$$
\begin{aligned}
\lambda \in \Lambda & =\left\{\boldsymbol{\lambda}: \boldsymbol{e}^{\prime} \boldsymbol{\lambda}=1, \boldsymbol{\lambda} \geq 0, \boldsymbol{\lambda} \in \Omega \subseteq \mathcal{R}_{+}^{m}\right\}, \\
\left(\overline{\boldsymbol{y}}_{i}, \Gamma_{i}\right) \in \mathcal{U}_{i} & \subseteq \mathcal{R}^{n} \times \mathcal{S}^{n}, i=1, \cdots, m
\end{aligned}
$$

where $\boldsymbol{e}=(1, \cdots, 1) \in \mathcal{R}^{m}, \Omega$ consists of some information on the distribution of exit time, and for each $i=1, \cdots, m, \mathcal{U}_{i}$ consists of all possible mean and covariance matrix of conditional distribution on exit time $\tau=t_{i}$, and there exists at least a pair $\left(\overline{\boldsymbol{y}}_{i}, \Gamma_{i}\right)$ in $\mathcal{U}_{i}$ such that $\Gamma_{i} \succ 0$. Then, the robust formation of portfolio selection with uncertain exit time is written as follows:

$$
\begin{aligned}
\min _{(\boldsymbol{x}, \gamma, \varsigma, \Delta) \in \mathcal{R}^{n} \times \mathcal{R} \times \mathcal{R} \times \mathcal{S}^{n}} & \gamma \\
\text { s.t. } \quad & \max _{\boldsymbol{\lambda} \in \Lambda} \max _{\left(\overline{\boldsymbol{y}}_{i}, \Gamma_{i}\right) \in \mathcal{U}_{i}} \sum_{i=1}^{m} \lambda_{i}\left[\left\langle\Delta, \Gamma_{i}\right\rangle-\boldsymbol{x}^{\prime} \overline{\boldsymbol{y}}_{i}\right]+\kappa(\epsilon)^{2} \varsigma \leq \gamma, \\
& \left(\begin{array}{cc}
\Delta & \boldsymbol{x} / 2 \\
\boldsymbol{x}^{\prime} / 2 & \varsigma
\end{array}\right) \succeq 0, \quad \boldsymbol{x} \in \mathcal{X} .
\end{aligned}
$$

Problem (6) is actually a min-max-max problem which is not easy to deal with immediately. To make it more tractable, we add some artificial variables and rewrite the problem as

$\left(P_{0}\right)$

$$
\begin{aligned}
\min _{(\boldsymbol{x}, \gamma, \varsigma, \Delta, \boldsymbol{\nu}) \in \mathcal{R}^{n} \times \mathcal{R} \times \mathcal{R} \times \mathcal{S}^{n} \times \mathcal{R}^{m}} & \gamma \\
\text { s.t. } \quad & \max _{\left(\overline{\boldsymbol{y}}_{i}, \Gamma_{i}\right) \in \mathcal{U}_{i}}\left\langle\Delta, \Gamma_{i}\right\rangle-\boldsymbol{x}^{\prime} \overline{\boldsymbol{y}}_{i} \leq \nu_{i}, i=1, \cdots, m, \\
& \max _{\boldsymbol{\lambda} \in \Lambda,} \boldsymbol{\lambda}^{\prime} \boldsymbol{\nu}+\kappa(\epsilon)^{2} \varsigma \leq \gamma, \\
& \left(\begin{array}{cc}
\Delta & \boldsymbol{x} / 2 \\
\boldsymbol{x}^{\prime} / 2 & \varsigma
\end{array}\right) \succeq 0, \quad \boldsymbol{x} \in \mathcal{X},
\end{aligned}
$$

where $\boldsymbol{\nu}=\left(\nu_{1}, \cdots, \nu_{m}\right)^{\prime}$. Clearly, if $\Lambda$ and $\mathcal{U}_{i}(i=1, \cdots, m)$ are compact, and $\mathcal{X}$ is convex, then $\left(P_{0}\right)$ is a convex programming problem. However, $\left(P_{0}\right)$ is not ready for application because of the "max" operation involved in the constraints. In the following, we show that, under some special structure of the set of parameters, it can be cast as a tractable convex programming problem, such as a semidefinite program.

\subsection{Uncertainty on conditional return distribution}

For simplicity, we consider the case where the moment pair $\left(\overline{\boldsymbol{y}}_{i}, \Gamma_{i}\right)$ is only known to belong to a given polytope for each exit time $t_{i}(i=1, \cdots, m)$. Mathematically, $\mathcal{U}_{i}$ is the convex hull of the vertices $\left(\overline{\boldsymbol{y}}_{i}^{1}, \Gamma_{i}^{1}\right), \cdots,\left(\overline{\boldsymbol{y}}_{i}^{L_{i}}, \Gamma_{i}^{L_{i}}\right)$, i.e.,

$$
\mathcal{U}_{i}=\mathbf{C o}\left\{\left(\overline{\boldsymbol{y}}_{i}^{1}, \Gamma_{i}^{1}\right), \cdots,\left(\overline{\boldsymbol{y}}_{i}^{L_{i}}, \Gamma_{i}^{L_{i}}\right)\right\}
$$

where $\left(\overline{\boldsymbol{y}}_{i}^{l}, \Gamma_{i}^{l}\right)\left(l=1, \cdots, L_{i}\right)$ are given. Notice that in (8) we do not limit the conditional distribution of portfolio exiting at time $t_{i}$ to be normally distributed. To be more specific, we assume that the polytopic uncertainty of mean and covariance matrix is separable, i.e.,

$$
\mathcal{U}_{i}=\mathcal{U}_{i}\left(\overline{\boldsymbol{y}}_{i}\right) \times \mathcal{U}_{i}\left(\Gamma_{i}\right)
$$


where $\mathcal{U}_{i}\left(\overline{\boldsymbol{y}}_{i}\right)=\mathbf{C o}\left\{\overline{\boldsymbol{y}}_{i}^{1}, \cdots, \overline{\boldsymbol{y}}_{i}^{L_{i}}\right\}$ and $\mathcal{U}_{i}\left(\Gamma_{i}\right)=\mathbf{C o}\left\{\Gamma_{i}^{1}, \ldots, \Gamma_{i}^{L_{i}}\right\}$. Then, for each $i=1, \cdots, m$, the left-hand side of constraint $(7)$ in $\left(P_{0}\right)$ can be written as

$$
\begin{array}{ll} 
& \max _{\left(\overline{\boldsymbol{y}}_{i}, \Gamma_{i}\right) \in \mathcal{U}_{i}}\left\langle\Delta, \Gamma_{i}\right\rangle-\boldsymbol{x}^{\prime} \overline{\boldsymbol{y}}_{i}=\max _{1 \leq l \leq L_{i}}\left\langle\Delta, \Gamma_{i}^{l}\right\rangle-\min _{1 \leq l \leq L_{i}} \boldsymbol{x}^{\prime} \overline{\boldsymbol{y}}_{i}^{l} \\
= & \min \left\{\alpha_{i}-\beta_{i}:\left\langle\Delta, \Gamma_{i}^{l}\right\rangle \leq \alpha_{i}, \beta_{i} \leq \boldsymbol{x}^{\prime} \overline{\boldsymbol{y}}_{i}^{l}, l=1, \cdots, L_{i}\right\}
\end{array}
$$

Thus, the operator "max" in constraint (7) can be replaced by (9) where "min" will be incorporated into the objective function of problem $\left(P_{0}\right)$. Consequently, we obtain the following minimization problem over $(\boldsymbol{x}, \gamma, \varsigma, \Delta, \boldsymbol{\nu}, \boldsymbol{\alpha}, \boldsymbol{\beta}) \in \mathcal{R}^{n} \times \mathcal{R} \times \mathcal{R} \times \mathcal{S}^{n} \times \mathcal{R}^{m} \times \mathcal{R}^{m} \times \mathcal{R}^{m}:$

$$
\begin{aligned}
\left(P_{1}\right) \min \quad & \gamma \\
\text { s.t. } \quad & \boldsymbol{\alpha}-\boldsymbol{\beta} \leq \boldsymbol{\nu}, \\
& \left\langle\Delta, \Gamma_{i}^{l}\right\rangle \leq \alpha_{i}, \quad l=1, \cdots, L_{i}, i=1, \cdots, m, \\
& \beta_{i} \leq \boldsymbol{x}^{\prime} \overline{\boldsymbol{y}}_{i}^{l}, l=1, \cdots, L_{i}, i=1, \cdots, m, \\
& \max _{\boldsymbol{\lambda} \in \Lambda,} \boldsymbol{\lambda}^{\prime} \boldsymbol{\nu}+\kappa(\epsilon)^{2} \varsigma \leq \gamma, \\
& \left(\begin{array}{cc}
\Delta & \boldsymbol{x} / 2 \\
\boldsymbol{x}^{\prime} / 2 & \varsigma
\end{array}\right) \succeq 0, \quad \boldsymbol{x} \in \mathcal{X},
\end{aligned}
$$

where $\boldsymbol{\alpha}=\left(\alpha_{1}, \cdots, \alpha_{m}\right)^{\prime}$ and $\boldsymbol{\beta}=\left(\beta_{1}, \cdots, \beta_{m}\right)^{\prime}$.

Now we are in a position to establish the following proposition:

Proposition 1 If $\left(\boldsymbol{x}^{*}, \gamma^{*}, \varsigma^{*}, \Delta^{*}, \boldsymbol{\nu}^{*}, \boldsymbol{\alpha}^{*}, \boldsymbol{\beta}^{*}\right)$ solves problem $\left(P_{1}\right)$, then $\boldsymbol{\alpha}^{*}-\boldsymbol{\beta}^{*}=\boldsymbol{\nu}^{*}$, and $\left(\boldsymbol{x}^{*}, \gamma^{*}, \varsigma^{*}, \Delta^{*}\right.$, $\left.\boldsymbol{\nu}^{*}\right)$ solves problem $\left(P_{0}\right)$. Conversely, if $(\overline{\boldsymbol{x}}, \bar{\gamma}, \bar{\zeta}, \bar{\Delta}, \overline{\boldsymbol{\nu}})$ solves problem $\left(P_{0}\right)$, then $(\overline{\boldsymbol{x}}, \bar{\gamma}, \bar{\zeta}, \bar{\Delta}, \overline{\boldsymbol{\nu}}, \overline{\boldsymbol{\alpha}}, \overline{\boldsymbol{\beta}})$ solves problem $\left(P_{1}\right)$, where $(\overline{\boldsymbol{\alpha}}, \overline{\boldsymbol{\beta}})$ is an optimal solution to $(9)$ with $(\boldsymbol{x}, \Delta)=(\overline{\boldsymbol{x}}, \bar{\Delta})$, and moreover $\overline{\boldsymbol{\alpha}}-\overline{\boldsymbol{\beta}}=\overline{\boldsymbol{\nu}}$.

It should be mentioned that there are various other approaches to specify the structure of $\mathcal{U}_{i}$ in the literature, and one can refer to El Ghaoui et al. (2003), Goldfarb and Iyengar (2003) and references therein for details.

\section{$3.2 \quad$ Uncertainty on exit time}

In this subsection, we discuss the distribution of exit time under the componentwise bounded uncertainty and semi-ellipsoidal uncertainty, individually.

\subsubsection{Componentwise bounded uncertainty}

Suppose $\Lambda$ in (10) is given as a componentwise bounded set such that

$$
\Lambda_{B}=\left\{\boldsymbol{\lambda}: \boldsymbol{e}^{\prime} \boldsymbol{\lambda}=1, \underline{\boldsymbol{\lambda}} \leq \boldsymbol{\lambda} \leq \overline{\boldsymbol{\lambda}}\right\},
$$

where $\underline{\boldsymbol{\lambda}}$ and $\overline{\boldsymbol{\lambda}}$ are two given constant vectors. The condition $\mathbf{e}^{\prime} \boldsymbol{\lambda}=1$ ensures $\boldsymbol{\lambda}$ to be a probability distribution, and the non-negativity constraint $\boldsymbol{\lambda} \geq 0$ is included in the bound constraints $\underline{\boldsymbol{\lambda}} \leq \boldsymbol{\lambda} \leq \overline{\boldsymbol{\lambda}}$. Since $\Lambda_{B}$ can be easily specified and reformulated in a tractable way, it is one of the most often used uncertain sets in robust optimization formulation.

Consider the following linear program:

$$
\begin{aligned}
\max _{\boldsymbol{\lambda} \in \mathcal{R}^{m}} & \boldsymbol{\nu}^{\prime} \boldsymbol{\lambda} \\
\text { s.t. } & \boldsymbol{e}^{\prime} \boldsymbol{\lambda}=1, \underline{\boldsymbol{\lambda}} \leq \boldsymbol{\lambda} \leq \overline{\boldsymbol{\lambda}} .
\end{aligned}
$$


We obtain the corresponding dual program as follows:

$$
\begin{array}{cl}
\min _{(z, \boldsymbol{\xi}, \boldsymbol{\omega}) \in \mathcal{R} \times \mathcal{R}^{m} \times \mathcal{R}^{m}} & z+\overline{\boldsymbol{\lambda}}^{\prime} \boldsymbol{\xi}+\underline{\boldsymbol{\lambda}}^{\prime} \boldsymbol{\omega} \\
\text { s.t. } & \boldsymbol{e} z+\boldsymbol{\xi}+\boldsymbol{\omega}=\boldsymbol{\nu}, \boldsymbol{\xi} \geq 0, \boldsymbol{\omega} \leq 0 .
\end{array}
$$

In relation to $\left(P_{1}\right)$, let us consider the following minimization problem over $(\boldsymbol{x}, \gamma, \varsigma, \Delta, \boldsymbol{\nu}, \boldsymbol{\alpha}$, $\boldsymbol{\beta}, z, \boldsymbol{\xi}, \boldsymbol{\omega}) \in \mathcal{R}^{n} \times \mathcal{R} \times \mathcal{R} \times \mathcal{S}^{n} \times \mathcal{R}^{m} \times \mathcal{R}^{m} \times \mathcal{R}^{m} \times \mathcal{R} \times \mathcal{R}^{m} \times \mathcal{R}^{m}:$

$$
\begin{aligned}
\left(P_{2}\right) \min \quad & \gamma \\
\text { s.t. } \quad & \boldsymbol{\alpha}-\boldsymbol{\beta} \leq \boldsymbol{\nu}, \\
& \left\langle\Delta, \Gamma_{i}^{l}\right\rangle \leq \alpha_{i}, l=1, \cdots, L_{i}, i=1, \cdots, m, \\
& \beta_{i} \leq \boldsymbol{x}^{\prime} \overline{\boldsymbol{y}}_{i}^{l}, \quad l=1, \cdots, L_{i}, i=1, \cdots, m, \\
& z+\overline{\boldsymbol{\lambda}}^{\prime} \boldsymbol{\xi}+\underline{\boldsymbol{\lambda}}^{\prime} \boldsymbol{\omega}+\kappa(\epsilon)^{2} \varsigma \leq \gamma, \\
& \boldsymbol{e} z+\boldsymbol{\xi}+\boldsymbol{\omega}=\boldsymbol{\nu}, \boldsymbol{\xi} \geq 0, \boldsymbol{\omega} \leq 0, \\
& \left(\begin{array}{cc}
\Delta & \boldsymbol{x} / 2 \\
\boldsymbol{x}^{\prime} / 2 & \varsigma
\end{array}\right) \succeq 0, \quad \boldsymbol{x} \in \mathcal{X} .
\end{aligned}
$$

Proposition 2 If $\left(\boldsymbol{x}^{*}, \gamma^{*}, \varsigma^{*}, \Delta^{*}, \boldsymbol{\nu}^{*}, \boldsymbol{\alpha}^{*}, \boldsymbol{\beta}^{*}, z^{*}, \boldsymbol{\xi}^{*}, \boldsymbol{\omega}^{*}\right)$ solves problem $\left(P_{2}\right)$, then $\left(\boldsymbol{x}^{*}, \gamma^{*}, \varsigma^{*}, \Delta^{*}, \boldsymbol{\nu}^{*}, \boldsymbol{\alpha}^{*}\right.$, $\left.\boldsymbol{\beta}^{*}\right)$ solves $\left(P_{1}\right)$. Conversely, if $(\overline{\boldsymbol{x}}, \bar{\gamma}, \bar{\varsigma}, \bar{\Delta}, \overline{\boldsymbol{\nu}}, \overline{\boldsymbol{\alpha}}, \overline{\boldsymbol{\beta}})$ solves problem $\left(P_{1}\right)$, then $(\overline{\boldsymbol{x}}, \bar{\gamma}, \overline{\boldsymbol{\zeta}}, \bar{\Delta}, \overline{\boldsymbol{\nu}}, \overline{\boldsymbol{\alpha}}, \overline{\boldsymbol{\beta}}, \bar{z}, \overline{\boldsymbol{\xi}}, \overline{\boldsymbol{\omega}})$ solves problem $\left(P_{2}\right)$, where $(\bar{z}, \overline{\boldsymbol{\xi}}, \overline{\boldsymbol{\omega}})$ is an optimal solution to problem (12) with $\boldsymbol{\nu}=\overline{\boldsymbol{\nu}}$.

\subsubsection{Semi-ellipsoidal uncertainty}

Suppose $\boldsymbol{\lambda}$ belongs to a semi-ellipsoid, (From the perspective of robust optimization, we may assume that $\boldsymbol{\lambda}$ belongs to an ellipsoid, i.e., $\boldsymbol{\lambda} \in \bar{\Lambda}_{E}=\left\{\boldsymbol{\lambda}: \boldsymbol{\lambda}=\boldsymbol{\lambda}^{0}+A \eta,\|\boldsymbol{\eta}\| \leq 1\right\}$. However, to ensure $\boldsymbol{\lambda}$ to be a probability distribution, it should meet the requirements $\boldsymbol{e}^{\prime} A \boldsymbol{\eta}=0$ and $\boldsymbol{\lambda}^{0}+A \boldsymbol{\eta} \geq 0$, which in turn restricts $\boldsymbol{\lambda}$ within a semi-ellipsoid. In this sense, we call $\boldsymbol{\lambda}$ semi-ellipsoidal uncertainty.) i.e.,

$$
\Lambda_{E}=\left\{\boldsymbol{\lambda}: \boldsymbol{\lambda}=\boldsymbol{\lambda}^{0}+A \boldsymbol{\eta}, \boldsymbol{e}^{\prime} A \boldsymbol{\eta}=0, \boldsymbol{\lambda}^{0}+A \boldsymbol{\eta} \geq 0,\|\boldsymbol{\eta}\|_{2} \leq 1\right\},
$$

where $\boldsymbol{e}^{\prime} \boldsymbol{\lambda}^{0}=1$ and $\boldsymbol{\lambda}^{0} \geq 0$, i.e., $\boldsymbol{\lambda}^{0}$ is a nominal distribution that is the center of the ellipsoid, and $A \in \mathcal{S}^{m}$ is the scaling matrix of the ellipsoid.

Consider the following convex program

$$
\begin{aligned}
& \max _{\boldsymbol{\eta} \in \mathcal{R}^{m}} \boldsymbol{\nu}^{\prime} A \boldsymbol{\eta} \\
& \text { s.t. } \quad \boldsymbol{e}^{\prime} A \boldsymbol{\eta}=0, \boldsymbol{\lambda}^{0}+A \boldsymbol{\eta} \geq 0,\|\boldsymbol{\eta}\|_{2} \leq 1 .
\end{aligned}
$$

The dual of (14) is the second-order cone program

$$
\begin{aligned}
& \min _{(\zeta, \boldsymbol{\omega}, \boldsymbol{\xi}, z) \in \mathcal{R} \times \mathcal{R}^{m} \times \mathcal{R}^{m} \times \mathcal{R}} \zeta+\left(\boldsymbol{\lambda}^{0}\right)^{\prime} \boldsymbol{\omega} \\
& \text { s.t. } \quad-\boldsymbol{\xi}-A \boldsymbol{\omega}+A^{\prime} \boldsymbol{e} z=A^{\prime} \boldsymbol{\nu},\|\boldsymbol{\xi}\|_{2} \leq \zeta, \boldsymbol{\omega} \geq 0 .
\end{aligned}
$$

Similarly to $\left(P_{2}\right)$, consider the following minimization problem over $(\boldsymbol{x}, \gamma, \varsigma, \Delta, \boldsymbol{\nu}, \boldsymbol{\alpha}, \boldsymbol{\beta}, z, \boldsymbol{\xi}, \boldsymbol{\omega}, \zeta) \in \mathcal{R}^{n} \times \mathcal{R} \times$ 
$\mathcal{R} \times \mathcal{S}^{n} \times \mathcal{R}^{m} \times \mathcal{R}^{m} \times \mathcal{R}^{m} \times \mathcal{R} \times \mathcal{R}^{m} \times \mathcal{R}^{m} \times \mathcal{R}$

$$
\begin{aligned}
\left(P_{3}\right) \min \quad & \gamma \\
\text { s.t. } \quad & \boldsymbol{\alpha}-\boldsymbol{\beta} \leq \boldsymbol{\nu}, \\
& \left\langle\Delta, \Gamma_{i}^{l}\right\rangle \leq \alpha_{i}, l=1, \cdots, L_{i}, i=1, \cdots, m, \\
& \beta_{i} \leq \boldsymbol{x}^{\prime} \overline{\boldsymbol{y}}_{i}^{l}, l=1, \cdots, L_{i}, i=1, \cdots, m, \\
& \left(\boldsymbol{\lambda}^{0}\right)^{\prime} \boldsymbol{\nu}+\zeta+\left(\boldsymbol{\lambda}^{0}\right)^{\prime} \boldsymbol{\omega}+\kappa(\epsilon)^{2} \varsigma \leq \gamma, \\
& -\boldsymbol{\xi}-A \boldsymbol{\omega}+A^{\prime} \boldsymbol{e} z=A^{\prime} \boldsymbol{\nu},\|\boldsymbol{\xi}\|_{2} \leq \zeta, \boldsymbol{\omega} \geq 0, \\
& \left(\begin{array}{cc}
\Delta & \boldsymbol{x} / 2 \\
\boldsymbol{x}^{\prime} / 2 & \varsigma
\end{array}\right) \succeq 0, \quad \boldsymbol{x} \in \mathcal{X} .
\end{aligned}
$$

Proposition 3 If $\left(\boldsymbol{x}^{*}, \gamma^{*}, \varsigma^{*}, \Delta^{*}, \boldsymbol{\nu}^{*}, \boldsymbol{\alpha}^{*}, \boldsymbol{\beta}^{*}, z^{*}, \boldsymbol{\xi}^{*}, \boldsymbol{\omega}^{*}, \zeta^{*}\right)$ solves problem $\left(P_{3}\right)$, then $\left(\boldsymbol{x}^{*}, \gamma^{*}, \varsigma^{*}, \Delta^{*}, \boldsymbol{\nu}^{*}\right.$, $\left.\boldsymbol{\alpha}^{*}, \boldsymbol{\beta}^{*}\right)$ solves $\left(P_{1}\right)$. Conversely, if $(\overline{\boldsymbol{x}}, \bar{\gamma}, \bar{\zeta}, \bar{\Delta}, \overline{\boldsymbol{\nu}}, \overline{\boldsymbol{\alpha}}, \overline{\boldsymbol{\beta}})$ solves problem $\left(P_{1}\right)$, then $(\overline{\boldsymbol{x}}, \bar{\gamma}, \bar{\zeta}, \bar{\Delta}, \overline{\boldsymbol{\nu}}, \overline{\boldsymbol{\alpha}}, \overline{\boldsymbol{\beta}}, \bar{z}, \overline{\boldsymbol{\xi}}, \overline{\boldsymbol{\omega}}, \bar{\zeta})$ solves problem $\left(P_{3}\right)$, where $(\bar{z}, \overline{\boldsymbol{\xi}}, \overline{\boldsymbol{\omega}}, \bar{\zeta})$ is an optimal solution to problem (15) with $\boldsymbol{\nu}=\overline{\boldsymbol{\nu}}$.

\section{Robust Portfolio Selection}

In this section we consider the situation that random returns of risky assets are just specified by a set of exit time distributions and a set of conditional return distributions on exit time, and formulate the portfolio management problem by adopting WVaR as the measure of risk.

Let $\mu$ be the worst-case minimum expected return required by the investor. Mathematically, this can be expressed as

$$
\min _{\boldsymbol{\lambda} \in \Lambda} \sum_{i=1}^{m}\left[\min _{\overline{\boldsymbol{y}}_{i} \in \mathcal{U}_{i}\left(\overline{\boldsymbol{y}}_{i}\right)} \lambda_{i}\left(\boldsymbol{x}^{\prime} \overline{\boldsymbol{y}}_{i}\right)\right] \geq \mu .
$$

Clearly, the worst-case minimum expected return involves two types of uncertainties, the uncertainty in the exit time distribution and the uncertainty in the expected return conditional on exit time.

In relation to (9), the left-hand side of (16) is equivalent to

$$
\begin{array}{ll}
\min _{\boldsymbol{\lambda} \in \Lambda} & \sum_{i=1}^{m} \lambda_{i} \beta_{i} \\
\text { s.t. } & \beta_{i}=\min _{1 \leq l \leq L_{i}} \boldsymbol{x}^{\prime} \overline{\boldsymbol{y}}_{i}^{l}, i=1, \cdots, m .
\end{array}
$$

Therefore, the feasible portfolio set $\mathcal{X}$ is specified as

$$
\left\{\boldsymbol{x}: \boldsymbol{e}^{\prime} \boldsymbol{x}=1, \underline{\boldsymbol{x}} \leq \boldsymbol{x} \leq \overline{\boldsymbol{x}}, \min _{\boldsymbol{\lambda} \in \Lambda} \boldsymbol{\beta}^{\prime} \boldsymbol{\lambda} \geq \mu, \beta_{i}=\min _{1 \leq l \leq L_{i}} \boldsymbol{x}^{\prime} \overline{\boldsymbol{y}}_{i}^{l}, i=1, \cdots, m\right\} .
$$

where $\boldsymbol{e}^{\prime} \boldsymbol{x}=1$ is the budget constraint and the inequalities $\underline{\boldsymbol{x}} \leq \boldsymbol{x} \leq \overline{\boldsymbol{x}}$ ensure diversification of an optimal portfolio with given lower and upper bounds $\underline{\boldsymbol{x}}$ and $\overline{\boldsymbol{x}}$.

\subsection{Portfolio formulation with componentwise bounded uncertainty on exit time}

In the case of componentwise bounded uncertainty in discrete distribution of exit time, by (11), the dual problem of the linear program involved in (17), i.e.,

$$
\begin{array}{cl}
\min _{\boldsymbol{\lambda} \in \mathcal{R}^{m}} & \boldsymbol{\beta}^{\prime} \boldsymbol{\lambda} \\
\text { s.t. } & \boldsymbol{e}^{\prime} \boldsymbol{\lambda}=1, \underline{\boldsymbol{\lambda}} \leq \boldsymbol{\lambda} \leq \overline{\boldsymbol{\lambda}},
\end{array}
$$


is expressed as

$$
\begin{array}{cl}
\max _{(\delta, \boldsymbol{\rho}, \boldsymbol{v}) \in \mathcal{R} \times \mathcal{R}^{m} \times \mathcal{R}^{m}} & \delta+\overline{\boldsymbol{\lambda}}^{\prime} \boldsymbol{\rho}+\underline{\boldsymbol{\lambda}}^{\prime} \boldsymbol{v} \\
\text { s.t. } & \boldsymbol{e} \delta+\boldsymbol{\rho}+\boldsymbol{v}=\boldsymbol{\beta}, \boldsymbol{\rho} \leq 0, \boldsymbol{v} \geq 0 .
\end{array}
$$

By the duality theory of linear programming, the optimal objective value of (19) gives a lower bound of the optimal value of problem (18). Moreover, if both the primal problem (18) and the dual problem (19) have optimal solutions, then the duality gap is zero. Therefore, it can be easily verified that $\mathcal{X}$ coincides with the set $\mathcal{X}_{B}$, which is expressed as

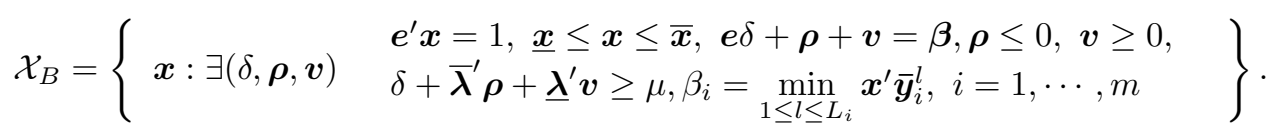

Thus, along with $\left(P_{2}\right)$ and Proposition 2, the robust portfolio selection problem with componentwise bounded uncertainty on exit time can be formulated as the following semidefinite program with variables $(\boldsymbol{x}, \gamma, \varsigma, \Delta, \boldsymbol{\alpha}, \boldsymbol{\beta}, z, \boldsymbol{\xi}, \boldsymbol{\omega}, \delta, \boldsymbol{\rho}, \boldsymbol{v}) \in \mathcal{R}^{n} \times \mathcal{R} \times \mathcal{R} \times \mathcal{S}^{n} \times \mathcal{R}^{m} \times \mathcal{R}^{m} \times \mathcal{R} \times \mathcal{R}^{m} \times \mathcal{R}^{m} \times \mathcal{R} \times \mathcal{R}^{m} \times \mathcal{R}^{m}:$

$$
\begin{aligned}
& \left(P_{4}\right) \quad \min \quad \gamma \\
& \text { s.t. } \quad \boldsymbol{e}^{\prime} \boldsymbol{x}=1 \text {, } \\
& \underline{\boldsymbol{x}} \leq \boldsymbol{x} \leq \overline{\boldsymbol{x}}, \\
& \boldsymbol{e} \delta+\boldsymbol{\rho}+\boldsymbol{v}=\boldsymbol{\beta}, \\
& \boldsymbol{\rho} \leq 0, \boldsymbol{v} \geq 0 \text {, } \\
& \delta+\overline{\boldsymbol{\lambda}}^{\prime} \boldsymbol{\rho}+\underline{\boldsymbol{\lambda}}^{\prime} \boldsymbol{v} \geq \mu, \\
& z+\overline{\boldsymbol{\lambda}}^{\prime} \boldsymbol{\xi}+\underline{\boldsymbol{\lambda}}^{\prime} \boldsymbol{\omega}+\kappa(\epsilon)^{2} \varsigma \leq \gamma, \\
& \boldsymbol{e} z+\boldsymbol{\xi}+\boldsymbol{\omega}=\boldsymbol{\alpha}-\boldsymbol{\beta}, \\
& \boldsymbol{\xi} \geq 0, \boldsymbol{\omega} \leq 0 \\
& \left(\begin{array}{cc}
\Delta & \boldsymbol{x} / 2 \\
\boldsymbol{x}^{\prime} / 2 & \varsigma
\end{array}\right) \succeq 0 \\
& \beta_{i} \leq \boldsymbol{x}^{\prime} \overline{\boldsymbol{y}}_{i}^{l} \\
& \left\langle\Delta, \Gamma_{i}^{l}\right\rangle \leq \alpha_{i}, l=1, \cdots, L_{i}, i=1, \cdots, m .
\end{aligned}
$$

It can be seen that the equalities $\beta_{i}=\min _{1 \leq l \leq L_{i}} \boldsymbol{x}^{\prime} \overline{\boldsymbol{y}}_{i}^{l}$ hold at optimality.

\subsection{Portfolio formulation with semi-ellipsoidal uncertainty on exit time}

In the case of semi-ellipsoidal uncertainty in distribution of exit time, by (13) and (17), $\mathcal{X}$ is given by

$$
\left\{\begin{array}{l}
\boldsymbol{e}^{\prime} \boldsymbol{x}=1, \underline{\boldsymbol{x}} \leq \boldsymbol{x} \leq \overline{\boldsymbol{x}}, \beta_{i}=\min _{1 \leq l \leq L_{i}} \boldsymbol{x}^{\prime} \overline{\boldsymbol{y}}_{i}^{l}, i=1, \cdots, m, \\
\boldsymbol{x}: \min _{\left\{\boldsymbol{\eta}: \mathbf{e}^{\prime} A \boldsymbol{\eta}=0, \boldsymbol{\lambda}^{0}+A \boldsymbol{\eta} \geq 0,\|\boldsymbol{\eta}\|_{2} \leq 1\right\}} \boldsymbol{\beta}^{\prime} A \boldsymbol{\eta} \geq \mu
\end{array}\right\} .
$$

Since the dual program of the second-order cone program

$$
\begin{aligned}
& \min _{\boldsymbol{\eta} \in \mathcal{R}^{m}} \boldsymbol{\beta}^{\prime} A \boldsymbol{\eta} \\
& \text { s.t. } \quad \boldsymbol{e}^{\prime} A \boldsymbol{\eta}=0, \quad \boldsymbol{\lambda}^{0}+A \boldsymbol{\eta} \geq 0,\|\boldsymbol{\eta}\|_{2} \leq 1
\end{aligned}
$$

is given as

$$
\begin{aligned}
& \max _{(\varrho, \boldsymbol{\rho}, \boldsymbol{v}, \delta) \in \mathcal{R} \times \mathcal{R}^{m} \times \mathcal{R}^{m} \times \mathcal{R}}-\varrho-\left(\boldsymbol{\lambda}^{0}\right)^{\prime} \boldsymbol{\rho} \\
& \text { s.t. } \quad \boldsymbol{v}+A^{\prime} \boldsymbol{\rho}+A^{\prime} \boldsymbol{e} \delta=A^{\prime} \boldsymbol{\beta},\|\boldsymbol{v}\|_{2} \leq \varrho, \boldsymbol{\rho} \geq 0,
\end{aligned}
$$


by the duality theory of second-order cone program, the optimal objective value of (21) gives a lower bound of the optimal value of problem (20). Moreover, if both the primal problem (20) and the dual problem (21) have optimal solutions, then the duality gap is zero. Therefore, it can be easily verified that $\mathcal{X}$ coincides with the following set $\mathcal{X}_{E}$, which is expressed as

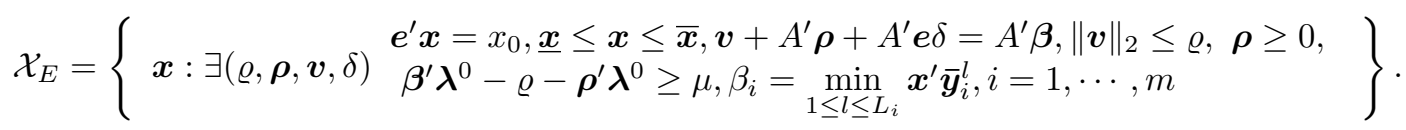

Now, together with $\left(P_{3}\right)$ and Proposition 3, the robust portfolio selection problem with semi-ellipsoidal uncertainty on exit time can be formulated as the following semidefinite program with variables $(\boldsymbol{x}, \gamma, \varsigma, \Delta, \boldsymbol{\alpha}, \boldsymbol{\beta}$, $z, \boldsymbol{\xi}, \boldsymbol{\omega}, \zeta, \varrho, \boldsymbol{\rho}, \boldsymbol{v}, \delta) \in \mathcal{R}^{n} \times \mathcal{R} \times \mathcal{R} \times \mathcal{S}^{n} \times \mathcal{R}^{m} \times \mathcal{R}^{m} \times \mathcal{R} \times \mathcal{R}^{m} \times \mathcal{R}^{m} \times \mathcal{R} \times \mathcal{R} \times \mathcal{R}^{m} \times \mathcal{R}^{m} \times \mathcal{R}:$

$$
\begin{aligned}
\left(P_{5}\right) \quad \min \quad & \gamma \\
\text { s.t. } \quad & \boldsymbol{e}^{\prime} \boldsymbol{x}=1, \\
& \underline{\boldsymbol{x}} \leq \boldsymbol{x} \leq \overline{\boldsymbol{x}}, \\
& \boldsymbol{v}+A^{\prime} \boldsymbol{\rho}+A^{\prime} \boldsymbol{e} \delta=A^{\prime} \boldsymbol{\beta}, \\
& \|\boldsymbol{v}\| \leq \varrho, \boldsymbol{\rho} \geq 0, \\
& \boldsymbol{\beta}^{\prime} \boldsymbol{\lambda}^{0}-\varrho-\boldsymbol{\rho}^{\prime} \boldsymbol{\lambda}^{0} \geq \mu, \\
& \left(\boldsymbol{\lambda}^{0}\right)^{\prime}(\boldsymbol{\alpha}-\boldsymbol{\beta})+\zeta+\left(\boldsymbol{\lambda}^{0}\right)^{\prime} \boldsymbol{\omega}+\kappa(\epsilon)^{2} \varsigma \leq \gamma, \\
& -\boldsymbol{\xi}-A \boldsymbol{\omega}+A^{\prime} \boldsymbol{e} z=A^{\prime}(\boldsymbol{\alpha}-\boldsymbol{\beta}), \\
& \|\boldsymbol{\xi}\|_{2} \leq \zeta, \boldsymbol{\omega} \geq 0 \\
& \left(\begin{array}{c}
\Delta \quad \boldsymbol{x} / 2 \\
\boldsymbol{x}^{\prime} / 2 \quad \varsigma
\end{array}\right) \succeq 0, \\
& \beta_{i} \leq \boldsymbol{x}^{\prime} \overline{\boldsymbol{y}}_{i}^{l}, \\
& \left\langle\Delta, \Gamma_{i}^{l}\right\rangle \leq \alpha_{i}, l=1, \cdots, L_{i}, i=1, \cdots, m .
\end{aligned}
$$

Similarly to the previous case, the equalities $\beta_{i}=\min _{1 \leq l \leq L_{i}} \boldsymbol{x}^{\prime} \overline{\boldsymbol{y}}_{i}^{l}$ hold at optimality.

\section{$5 \quad$ Numerical Experiments}

In this section, we present a simple but illustrative numerical experiment with the robust portfolio selection problem involving an uncertain exit time. Without loss of generality, we only consider the case of componentwise bounded uncertainty. The reason is that a nonempty box must contain a smaller semi-ellipsoid and at the same time must be contained by a larger semi-ellipsoid. Therefore, for both the componentwise bounded and semi-ellipsoidal uncertainties, the results will be similar. Moreover, as mentioned in Section 3, componentwise bounded uncertainty is one of the most often used uncertain sets in robust optimization.

We take the example given by Huang et al. (2006), where the portfolio to be constructed consists of 10 stocks from the Tokyo Stock Exchange. Suppose that the investment horizon is two days, and that the investor may exit market at the end of the first day, i.e., $m=2$. Because of inconsistent behavior of returns among different time periods, we divide the return series into two sub-samples, i.e., $L_{i}=2(i=1,2)$, and then estimate the corresponding expected returns and covariance matrices, which are regarded as the scenarios of the conditional distribution of return. (We collect the historical daily closing prices of these stocks from January 19, 1994 to December 31, 2004, which includes 2701 samples. For the return computations of one day and two days, we use $y_{t}^{1}=\frac{V_{t}-V_{t-1}}{V_{t-1}} \times 100, t=2,3,4, \ldots$ and $y_{t}^{2}=\frac{V_{t}-V_{t-2}}{V_{t-2}} \times 100, t=3,5,7, \ldots$, where $V_{t}$ denotes the closing price of stock at the day $t$, see Huang et al. (2006) for details.) Thus, we obtain $\left(\overline{\boldsymbol{y}}_{i}^{l}, \Gamma_{i}^{l}\right), l=1,2, i=1,2$. In this example, we set $\underline{\boldsymbol{x}}=0, \overline{\boldsymbol{x}}=\boldsymbol{e}$. Let WVaR-I denote the original WVaR given in El Ghaoui (2003), where the investor terminates his portfolio at the end of investment horizon, and let WVaR-II denote our model which incorporates the uncertainty in exit time. Numerical experiments for 
WVaR-I and WVaR-II are performed using the semidefinite programming formulation $\left(P_{4}\right)$. (We solve the problem by Matlab701 and SeDuMi1.05 (Sturm, 2001) on PC with Intel Pentium 4 CPU 3.00GHz, 1.5GB RAM.) In the computation of WVaR-I, we set $\underline{\lambda}_{1}=\bar{\lambda}_{1}=0$ and $\underline{\lambda}_{2}=\bar{\lambda}_{2}=1$.

Table 1: Comparison of performances of WVaR-I and WVaR-II.

\begin{tabular}{|c|c|c|c|c|c|}
\hline \multirow{2}{*}{$\begin{array}{c}\mu \\
(\%)\end{array}$} & \multirow{2}{*}{$\begin{array}{c}\underline{\boldsymbol{\lambda}}=0, \overline{\boldsymbol{\lambda}}=\boldsymbol{e} \\
\epsilon=10 \%\end{array}$} & \multicolumn{2}{|c|}{ Mean (\%) } & \multicolumn{2}{|c|}{ WVaR (\%) } \\
\hline & & Exit time 1 & Exit time 2 & Exit time 1 & Exit time 2 \\
\hline \multirow{2}{*}{0.005} & WVaR-I & 0.0056 & 0.0050 & 3.8639 & 4.9755 \\
\hline & WVaR-II & 0.0056 & 0.0050 & 3.8639 & 4.9755 \\
\hline \multirow{2}{*}{0.010} & WVaR-I & 0.0083 & 0.0100 & 4.0025 & 5.1423 \\
\hline & WVaR-II & 0.0100 & 0.0129 & 4.1181 & 5.2704 \\
\hline \multirow{2}{*}{0.020} & WVaR-I & 0.0136 & 0.0200 & 4.4054 & 5.6239 \\
\hline & WVaR-II & 0.0200 & 0.0316 & 5.0651 & 6.4081 \\
\hline \multirow{2}{*}{0.025} & WVaR-I & 0.0163 & 0.0250 & 4.6582 & 5.9262 \\
\hline & WVaR-II & - & - & - & - \\
\hline
\end{tabular}

To compare the performance of the optimal portfolios resulting from WVaR-I and WVaR-II for various values of the required minimal worst-case expected return $\mu$, Table 1 shows the expected values and WVaRs when $\epsilon=0.1$. Actually, by $\left(P_{4}\right)$, we can compute the actual expected returns and corresponding WVaRs by means of the WVaR-I and WVaR-II models when the investor exits the market at different times. It is obvious that the larger the required minimal worst-case expected return, the larger the associated risk. Ostensibly, for the same $\mu$, the risk of WVaR-II appears to be larger than that of WVaR-I. However, higher risk is compensated by higher return. Moreover, the larger value of WVaR-II does not necessarily means higher risk than that of WVaR-I, which is only because the investor considers more seriously the uncertainty of future extreme scenarios and hence takes a conservative strategy. On the other hand, in the WVaRII model, the worst-case expected return can be guaranteed even if the investor terminates his portfolio before maturity. For example, when $\mu=0.01$, if the investor exits the market at the end of first day, the actual portfolio return resulting from WVaR-I is 0.0083 , which is less than the required minimal expected return $\mu=0.01$. In this case, the optimal portfolio of WVaR-II meets this requirement. Moreover, it will be easy to show quantitatively that WVaR-II is much preferred to WVaR-I in general if we define unit return-risk ratio as $L=$ (actual return)/risk. For example, if $\mu=0.020$ and the exit takes place at the first day, then $L(\mathrm{WVaR}-\mathrm{I})=0.0136 / 4.4054=0.0031$, while $L(\mathrm{WVaR}-\mathrm{II})=0.0200 / 5.0651=0.0039$, i.e., $L(\mathrm{WVaR}-\mathrm{I}) \leq$ $L($ WVaR-II), which implies that for the same risk, the investor considering the uncertain exit time may obtain more risk premium. This interesting phenomenon also applies to other cases $(\mu=0.01,0.02)$.

Table 2: WVaR-II for various pairs $\left(\underline{\lambda}_{1}, \bar{\lambda}_{1}\right)(\mu=0.02)$.

\begin{tabular}{cc|cccccc}
\hline \hline \multicolumn{1}{c}{$\underline{\lambda}_{1}$} & $\bar{\lambda}_{1}$ & 0.0 & 0.2 & 0.4 & 0.6 & 0.8 & 1 \\
\hline 0.0 & 5.6239 & 5.7052 & 5.8088 & 5.9449 & 6.1313 & 6.4081 \\
0.1 & - & 5.5808 & 5.6828 & 5.8167 & 5.9998 & 6.2723 \\
0.2 & - & 5.4536 & 5.5538 & 5.6854 & 5.8653 & 6.1335 \\
0.3 & - & - & 5.4216 & 5.5510 & 5.7275 & 5.9913 \\
0.4 & - & - & 5.2860 & 5.4131 & 5.5863 & 5.8456 \\
0.5 & - & - & - & 5.2715 & 5.4413 & 5.6961 \\
\hline \hline
\end{tabular}


To explore the impact of the uncertainty in exit time on WVaR, Table 2 reports the values of WVaR-II for vaious choices of $\underline{\boldsymbol{\lambda}}$ and $\overline{\boldsymbol{\lambda}}$. (In our example, $\underline{\lambda}_{2}=1-\bar{\lambda}_{1}$ and $\bar{\lambda}_{2}=1-\underline{\lambda}_{1}$, so we need only consider $\underline{\lambda}_{1}$ and $\bar{\lambda}_{1}$.) Obviously, for a fixed $\underline{\lambda}_{1}$ in Table 2, an increase of $\bar{\lambda}_{1}$ results in an increase of WVaR-II. On the contrary, WVaR-II decreases as $\underline{\lambda}_{1}$ increases. For example, when $\underline{\lambda}_{1}$ increases from 0 to 0.5 , WVaR-II decreases from 5.9449 to 5.2715 with fixed $\bar{\lambda}_{1}=0.6$. We can explain this phenomenon as follows: the uncertainty of exit time is dependent on the area of the uncertain set of $\boldsymbol{\lambda}$. The larger the area, the greater the uncertainty. This is because the area of $\boldsymbol{\lambda}$ equals $S_{\boldsymbol{\lambda}}=\left(\bar{\lambda}_{1}-\underline{\lambda}_{1}\right) \cdot\left(\bar{\lambda}_{2}-\underline{\lambda}_{2}\right)=\left(\bar{\lambda}_{1}-\underline{\lambda}_{1}\right)^{2}$. Thus, for fixed $\bar{\lambda}_{1}$, as $\underline{\lambda}_{1}$ increases, the interval of $\left[\underline{\lambda}_{1}, \bar{\lambda}_{1}\right]$ shrinks $\left(\bar{\lambda}_{1}-\underline{\lambda}_{1}\right.$ decreases $)$, which reduces the area of $\boldsymbol{\lambda}\left(\left(\bar{\lambda}_{1}-\underline{\lambda}_{1}\right)^{2}\right.$ decreases $)$, and hence reduces the uncertainty of exit time, i.e., the distribution of exit time belongs to a smaller box, and hence, WVaR-II decreases.

\section{Conclusion}

The uncertainty in exit time and the uncertainty in the conditional distribution of portfolio on exit time give rise to an important and complicated problem in practice. In this paper, we extend the original WVaR approach developed by El Ghaoui et al. (2003) to combine the two types of uncertainties, and formulate the problem as a semidefinte program, which can be efficiently solved. A simple numerical experiment shows the usefulness of the proposed model.

\section{References}

[1] Bertsimas, D. and I. Popescu (2005) Optimal Inequalities in Probability Theory: A Convex Optimization Approach, SIAM Journal on Optimization, 15 (3), 780-804.

[2] Black, F., and R. Litterman (1992) Global Portfolio Optimization, Financial Analysts Journal, 48, $28-43$.

[3] Costa, O.L. and A.C. Paiva (2002) Robust Portfolio Selection Using Linear-Matrix Inequality, Journal of Economic Dynamics \& Control, 26, 889-909.

[4] El Ghaoui, L., Oks, M. and F. Oustry (2003) Worst-Case Value-at-Risk and Robust Portfolio Optimization: A Conic Programming Approach, Operations Research, 51, 543-556.

[5] Goldfarb, D. and G. Iyengar (2003) Robust Portfolio Selection Problems, Mathematics of Operations Research, 28, 1-38.

[6] Huang, D., Zhu, S.S., Fabozzi, F.J. and M. Fukushima (2005) Robust CVaR Approach to Portfolio Selection with Uncertain Exit Time, Technical Report 2006-1, Department of Applied Mathematics and Physics, Graduate School of Informatics, Kyoto University, http://www.amp.i.kyoto-u.ac.jp/tecrep/.

[7] Jorion, P. (2001) Value at Risk: the New Benchmark for Managing Financial Risk, McGraw-Hill, New York.

[8] Martellini, L. and B. Urošević (2005) Static Mean-Variance Analysis with Uncertain Time Horizon, forthcoming in Management Science.

[9] Sturm, J. (2001) Using SeDuMi, A Matlab Toolbox for Optimization over Symmetric Cones. Department of Econometrics, Tilburg University, The Netherlands.

[10] Zhu, S.S. and M. Fukushima (2005) Worst-Case Conditional Value-at-Risk with Application to Robust Portfolio Management, Technical Report 2005-6, Department of Applied Mathematics and Physics, Graduate School of Informatics, Kyoto University, http://www.amp.i.kyoto-u.ac.jp/tecrep/. 